\title{
Capabilities of Laser-Induced Marks as Information Carriers Created in Different Materials
}

\author{
Volodymyr A. Gnatyuk ${ }^{* 1}$, Oleksandr I. Vlasenko ${ }^{* 1}$, Sergiy N. Levytskyi ${ }^{* 1}$, Toru Aoki ${ }^{* 2,3}$, Vygantas Mizeikis ${ }^{* 3}$, \\ Sergey V. Gagarsky*4, Kateryna S. Zelenska*5 and Dmytro V. Gnatyuk ${ }^{* 6}$ \\ ${ }^{*}$ V.E. Lashkaryov Institute of Semiconductor Physics of the National Academy of Sciences of \\ Ukraine, 41 Nauky Ave., Kyiv 03028, Ukraine \\ E-mail: gnatyuk@ua.fm \\ ${ }^{* 2}$ Graduate School of Informatics, Shizuoka University, 3-5-1 Johoku, Naka-ku, \\ Hamamatsu 432-8011, Japan \\ ${ }^{* 3}$ Research Institute of Electronics, Shizuoka University, 3-5-1 Johoku, Naka-ku, \\ Hamamatsu 432-8011, Japan \\ ${ }^{*} 4$ ITMO University, 49 Kronverksky Ave., Saint Petersburg 197101, Russia \\ ${ }^{* 5}$ Taras Shevchenko National University of Kyiv, 64/13 Volodymyrska Str., Kyiv 01601, Ukraine \\ ${ }^{* 6}$ Graduate School of Science and Technology, Shizuoka University, 3-5-1 Johoku, Naka-ku, \\ Hamamatsu 432-8011, Japan
}

\begin{abstract}
Laser technological approaches and techniques suitable for volumetric optical recording of digital data and image formation by creation of micromarks (data bits, pixels, and other information carriers) in the bulk of different materials are analyzed. Laser-induced marking centers with different controlled geometric parameters (size, shape, orientation, etc.) are obtained by varying the focal area, wavelength, duration and energy of laser pulses, temperature of objects and also applying external or local internal stresses in irradiated samples. The laser-based methods for creation of colored and dark marks in transparent glasses and polymers with special light-absorbing inclusions are studied. Black marks are created in plastics because of formation of carbonaceous products as a result of pyrolysis of the polymer matrix due to absorption of focused laser radiation by optical inhomogeneities. Embedding absorptive microparticles into the volume of transparent polymers makes the marking procedure more effective and predicted and it increases contrast and resolution of the marking area inside the plastics. The laser-based techniques of formation of light-emitting centers in specialized fluorescent materials and their applicability for data recording are discussed. Emitting marks are obtained in thermally stable photosensitive organic chromone-based materials by twophoton interaction process under irradiation with a focused laser beam.
\end{abstract}

DOI: $10.2961 / \mathrm{jlmn} .2016 .02 .0005$

Keywords: laser-induced marks, absorbing centers, color marks, light-emitting centers, image pixel, information carrier, transparent dielectrics, multiphoton absorption, volumetric recording

\section{Introduction}

Short pulses of focused laser radiation have the unique capacity to change the properties of a small local area in the bulk of transparent objects because of non-ideal optical transparency of the employed material, its absorptive inhomogeneities (defects, inclusions, density fluctuations, etc.) and non-linear phenomena (multiphoton absorption, etc.) [1-6]. Regardless of the specific absorption mechanism occurring under high intensity laser radiation, a superfast heating takes place in the vicinity of the laser beam focal region which drastically heats to extremely high temperatures. Depending on the irradiated materials, this results in generation of high internal stresses with formation of a crack and/or void in solids, modification of the refractive index and structure in crystalline materials, redistribution of nanoparticles and microinclusions in composite compounds, change of the chemical composition in polymers up to creation of a burnt area, and also creation of light-emitting centers in fluorescent organic materials [6-
27]. A resulting local area with the optical properties different from those of the matrix (bulk), i.e. permanent microdefect (damage, crack, void, pore, etc.) can be considered as an information carrier or image pixel. Spatially controlled laser-induced volumetric formation of marking centers (information carriers) offers novel opportunities for creation of digital recording areas or images in a variety of materials and objects [8-10, 22-27].

In conventional optical recording methods, identical marks (point-like spots, round or elliptical pits, etc.) are used as elementary (one-bit) carriers. The capability of optical recording can be enhanced by creation of multi-bit carriers. The key problem is how to create not only elementary (one-bit) carriers, but multi-bit carriers using a laser beam focused inside the materials. This can be solved by formation of parameterized marks, i.e. laser-induced centers with controlled parameters (shape, size, orientation, density, reflectivity, absorptivity, color, emissivity, brightness, etc.). In practical applications, it will be desirable for 
laser-induced marks to be parameterized and created in such a way so that their shapes and other characteristics become easily distinguishable and thus directly usable in digital recording.

Some of the existing laser-based marking techniques are in principle suitable for creation of marks with various geometric parameters in transparent dielectrics [6-11, 1821]. However, the possibilities to control the characteristics of such marks, as discussed in the literature, are rather limited, the corresponding techniques have not been developed enough to be widely used in many practical applications, therefore the analysis of the promising methods and further experimental studies are necessary. Aside from their linear dimensions, shapes and orientations, marking centers can be also characterized and differ in brightness (darkness), color, light-emitting ability and other characteristics [1022]. Thus, it would be particularly interesting to explore some specialized methods for creation of dark (black) and colored marks, and light-emitting centers.

On the base of the experimental results and literature data on laser-induced formation of marks in various materials under different irradiation conditions, the laser techniques for creation of geometrically parameterized, colorenhanced, dark and light-emitting marking centers are discussed to develop new opportunities of efficient and reliable volumetric marking, formation of images and recording of digital information. We have evaluated various laserbased techniques of formation of parameterized micromarks in the bulk of different materials transparent for a selected laser wavelength, which can serve as information carriers (data bits, pixels, and others) for 2D and 3D optical recording of digital data or creation of images.

\section{Laser-induced microdamages in solids (glass) and creation of parameterized marks}

Several methods and laser-based techniques of formation of marks with predetermined geometric parameters (size, shape, orientation, density etc.) and morphology inside transparent solid-state materials particularly in glass have been discussed [6-12]. Different kinds of marks can be obtained by varying the laser focal area, wavelength, duration, and energy of laser pulses, temperature of the object and also applying general external or local internal compressive or tensile stresses in the irradiated object. However, possibilities for controlling the appearance of parameterized marks which are easily distinguishable and usable for information recording are very limited and not studied well.

Fig. 1(a) shows the schematic process of irradiation of a transparent sample with a focused laser beam to create a marking center (microcrack) in its volume. Marks with different sizes were formed under irradiation with a single pulses $(\tau=7 \mathrm{~ns})$ of the second harmonic $(\lambda=532 \mathrm{~nm})$ of a YAG:Nd laser with increasing output energy $E$ (Fig. 1(b)).

The oblong marks in the scaled-up lateral view of the glass sample (Fig. 1(b), B) were obtained by an astigmatically focused laser beam oriented vertically. Laser-induced extended microcracks in the ellipsoid-like shape focal area are oriented in the direction of the laser beam. The sizes of microcracks can be controlled up to a certain extent by adjustment of the length of the laser beam focal area. In this way it becomes possible to form dash-like marks with varied lengths [9-11].

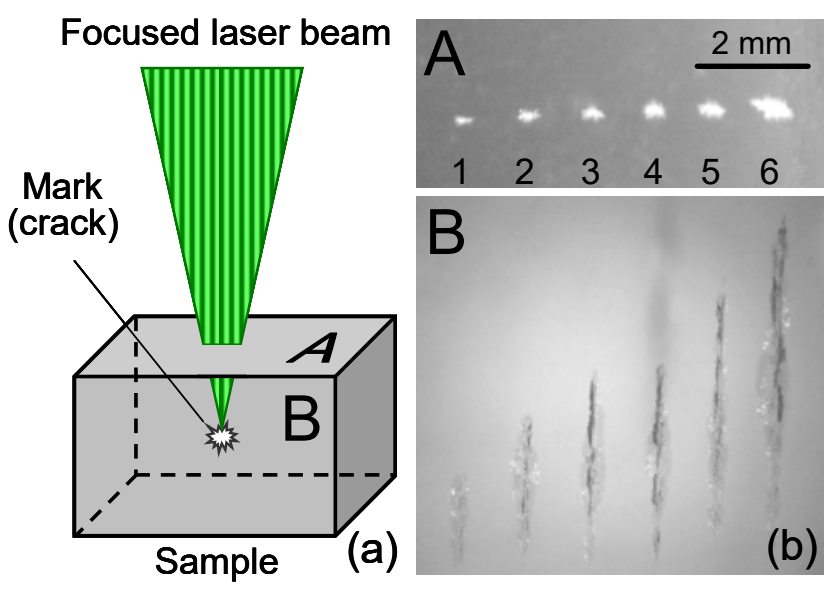

Fig. 1 The schematic image illustrating the procedure of laserinduced creation of a mark in the bulk of a sample which is transparent for the applied laser radiation (a) and micrographs of laserinduced marks formed inside the glass bulk by single laser pulses with different energy $E$. The micrographs (b) taken from the front

$\mathrm{A}$ and lateral $\mathrm{B}$ sides (a) show the corresponding view of mi-

crocracks with different diameters $d$ and lengths $l$ (Table 1).

The relationships between the geometrical parameters of marks and laser characteristics are shown in Table 1.

Table 1 Dependences of the sizes (diameters $d$ and lengths $l$ ) of the microcracks (Fig. 1(b)) on the output laser pulse energy $E$.

\begin{tabular}{|c|c|c|c|c|c|c|}
\hline No & 1 & 2 & 3 & 4 & 5 & 6 \\
\hline$E, \mathrm{~mJ}$ & 11.2 & 18.6 & 23.6 & 26.7 & 30.2 & 70.6 \\
\hline$d, \mathrm{~mm}$ & 0.19 & 0.25 & 0.26 & 0.28 & 0.40 & 0.53 \\
\hline$l, \mathrm{~mm}$ & 1.64 & 2.4 & 3.1 & 3.3 & 4.2 & 6.7 \\
\hline
\end{tabular}

Volumetric laser-induced marking in glass is realized by physical damage of the material and creation of a structural microdefect (crack, cavern, pore, void, etc.). (Figs. 1(b) and 2). The size of the cracked area usually much exceeds that of the focused laser beam spot which can be limited to several micrometers. Therefore, it is important to create marks (information carrier) with smaller sizes to decrease the distance between neighbour marks and achieve higher recording density of digital data. If marks are rather large and not properly spaced, this leads to merging of microcracks from adjacent marks (Fig. 2(a)).

For optimizing the morphology and decreasing the size of laser-induced marks, laser pulses with shorter wavelength $\lambda$ and duration $\tau$ were employed (Fig. 2(b)). To increase the spatial resolution, minimize the distortion and avoid marks overlapping, laser-induced marking was carried out in the glass preheated up to a softening state (Fig. 2(b)). With this method mark spacing can be reduced.

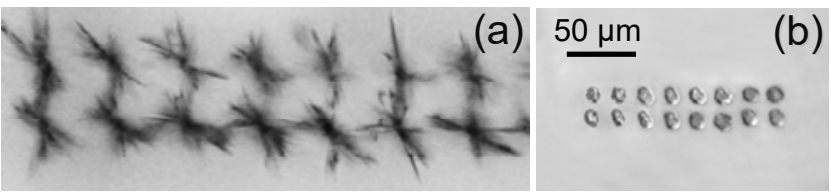

Fig. 2 Laser induced marks inside the glass bulk created under different laser irradiation conditions: wavelength $\lambda=532 \mathrm{~nm}$, pulse duration $\tau=7 \mathrm{~ns}$, temperature $T=20^{\circ} \mathrm{C}$ and distance between marks $a=60 \mu \mathrm{m}(\mathrm{a}) ; \lambda=355 \mathrm{~nm}, \tau=4.2 \mathrm{~ns}, T=600^{\circ} \mathrm{C}$ and $a=20 \mu \mathrm{m}$ (b). Magnification is the same in (a) and (b). 


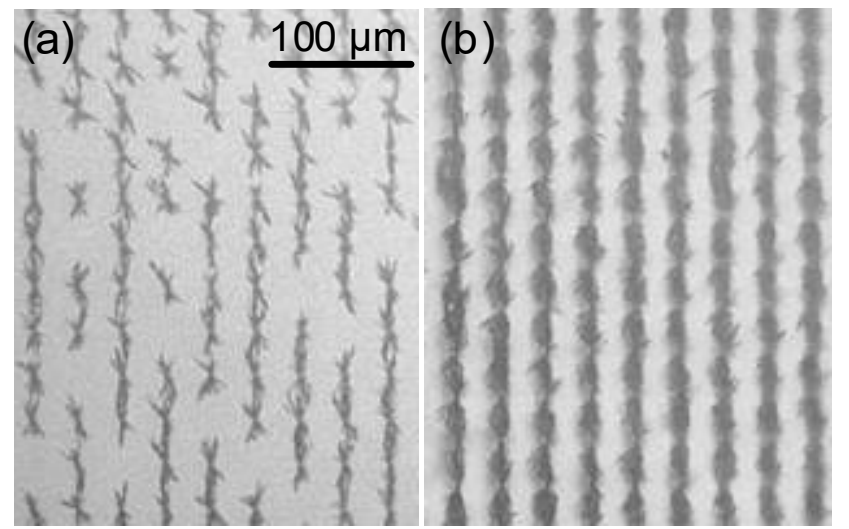

Fig. 3 Microphotographs of two areas inside the glass sample subjected to uniaxial stress under laser-induced marking with lower (a) and higher laser pulse energy (b).

Laser irradiation of an object subjected to uniaxial compression or tension can lead to appearance of dash-like cracks and line-like damages oriented along the stress gradient. In Fig. 3, the array of marks appears shown as vertical lines, the shape of each mark is elongated. The regions of the bulk glass shown in Fig. 3 were subjected to uniaxial deformation which created an internal stress field. This resulted the appearance of extended microcracks along the stress gradient under irradiation with laser pulses of lower (a) and higher (b) energy.

Thus, it is possible to control the length and orientation of such extended marks by respectively changing the magnitude and direction of the external stresses applied to the transparent object during its irradiation with laser pulses focused to a point at certain depth inside the material [9, 10]. The local compressive or tensile stresses can be preinduced with an additional $\mathrm{CW}$ or pulsed laser beam, spatially coinciding with the recording pulse.

Thus, laser pulses with longer wavelength and duration result bigger material damage and create larger microcracks. For really small marks, nano-, pico-, or femtosecond laser pulses of short wavelength (ultraviolet) should be employed [6-12]. The spatial resolution and density of created marks in glass objects can be increased by preheating the region where the volumetric recording area is formed to temperature close the material melting point [10].

\section{Laser-based methods and technologies for creation of colored marks in glass}

Various laser-based technologies for color laser marking in transparent materials have been investigated and their optimization for industrial applications have been considered. Some promising approaches for formation of colored centers by a focused laser beam in glasses with special inclusions are analyzed.

\subsection{Glasses doped with metal oxide atoms}

Modification of the oxidation state of metal ions in doped glasses in the laser focal region leads to formation of color centers (light absorption is changed by polyvalent ions) [13-15]. Note that color centers are formed through a mechanism that is different from microdamage so fractures in the glass are in fact undesirable and should be eliminated by an appropriate selection of the laser irradiation parame- ters. To form color marks, different types of lasers can be used with wavelength ranging from UV to IR and with pulse durations from femto- to tens of nanoseconds [14].

\subsection{Photosensitive glasses}

Color marks can be created in glasses doped with impurities based on $\mathrm{F}, \mathrm{Ag}, \mathrm{Br}$, and others which are sensitive to laser radiation. For example, silicate glasses with impurities of salts of $\mathrm{Na}, \mathrm{Ca}, \mathrm{Zn}$, and $\mathrm{Al}$ as well as glasses doped with $\mathrm{Ag}, \mathrm{Ce}$, and $\mathrm{F}$ change their complex refractive index after UV exposure and following thermal annealing [15-17]. A method for formation of latent (invisible) marks can therefore be implemented by employing laser pulses of different wavelengths and sufficiently high intensity leading to the generation of plasma and UV radiation in the focal area of the material. The resulting latent marks is not visible and will appear as colored marks only after thermal annealing of the laser-irradiated object.

Fig. 4 shows experimentally obtained brown-colored areas in the bulk of a special type of glass with embedded Ag-based photosensitive centers after irradiation with nanosecond pulses of an UV laser and following annealing. Single pulses $(\tau=20 \mathrm{~ns})$ of the $3 \mathrm{rd}$ harmonic $(\lambda=355 \mathrm{~nm})$ of a YAG:Nd laser were used. Irradiation was carried out by an unfocused laser beam with the diameter of $5 \mathrm{~mm}$ in air. The exposure (energy density integrated over time) was collected by a series of a few hundreds of laser pulses and the value was about $50 \mathrm{~J} / \mathrm{cm}^{2}$. The colored area with the size of a laser spot appeared after the corresponding steadystate annealing of the sample in a muffle furnace.

While obtained color marks are good for imaging, they appear to be too coarse for meaningful and efficient digital data recording. This might be due to the specific physical distribution of the photosensitive centers in the bulk of material. To clarify the issue, further studies would be required.

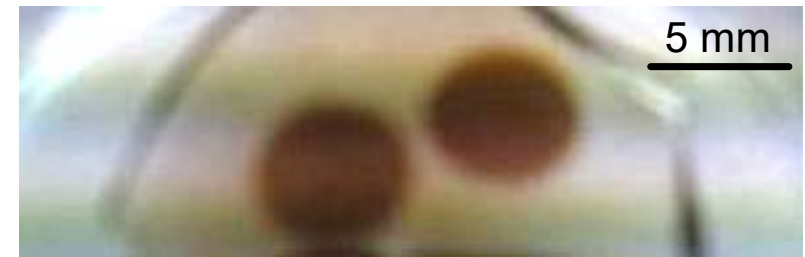

Fig 4 Colored areas formed by nanosecond UV laser pulses in the Ag-doped glass.

Fig. 4 demonstrates the maximally possible optical density (brown color) achieved in a single layer at the thickness of a few tens of microns inside the glass bulk. Perhaps, the discussed colored marking technique is not well suitable for bit-data recording because of energy inefficiency and impossibility to reach a small enough colored mark (pixel, data bit, i.e. information carrier). However the method of colored marking can be used for technological marking, creation of art items, etc.

\subsection{Transparent materials with colored impurities}

In the case of volumetric laser-induced marking of transparent materials containing colored impurity (microparticles acting as point defects), a mark (microcrack) created in the bulk of such material can act as extended structural microdefect having gettering properties with respect 
to point defects (colored micoparticles) [28]. Colored micoparticles move (diffuse) toward the created mark with high diffusion velocity as result of action of laser-generated high-gradient thermal and stress fields in the vicinity of the mark [5]. Moreover, accumulation of point defects (colored impurity) at extended defects (microcracks) can proceed after laser action as result of segregation of them in the residual stress field in the vicinity of an extended defect (mark). The segregation velocity depends on the stress gradient, characteristics of the material and point defects [28]. Thus, the concentration of colored impurity increases near the laser-induced crack and this area appears as the colored mark.

The initial concentration of a colored impurity in such materials is high, but it should be kept under the natural limit of color appearance to avoid seeing colorization. Laser-induced microdamage (mark) in the bulk of such materials, however, leads to apparent coloration due to the increased impurity density in result of laser-stimulated diffusion and segregation of color centers at that microdamage. Consequently, gettering properties of laser-induced microdamages with respect to colored impurity and velocities of diffusion and segregation of impurity particles under and after extreme conditions of irradiation define the color intensity and formation time of the colored marking area.

\section{Laser-induced marking in transparent plastic mate- rials and polymers with light-absorbing microparticles}

Plastic materials are suitable for creation of laserinduced dark or black marks. Dark local areas can be relatively easily formed inside polymers because focused laser irradiation changes the chemical composition of a molten (or even burned) area. We have investigated the dark spots in the centers of the volumetric marks created in commercial plastics and attributed them to charring of the materials [18]. The resulting process was refined for persistent laser marking of laminating films [10].

The observed carbonization reaction occurs when the absorbed laser radiation energy increases the temperature of the material surrounding the focal region to a level sufficient to incur degradation of the plastic. In presence of oxygen it leads to combustion of the polymer, but in case of limited access of oxygen, only charring of the plastic with formation of a dark mark occurs. However, in case of sufficiently high laser intensity, a void (pore) can be formed in the bulk of the plastic as a result of combustion of the material in the central part of the focal region [18].

Dark marks are created in plastics by carbonaceous products formed as a result of pyrolysis of the polymer matrix due to absorption of focused laser radiation by optical inhomogeneities including residual impurities or introduced microinclusions. Contrast and resolution of laser marking inside transparent plastic materials can be increased by embedding light-absorbing microparticles. In particular, volumetric formation of laser-induced marking centers in polymers can be enhanced by adding carbonbased microparticles [19-21]. It is generally accepted that carbon-based objects can be considered as a black body [19-21, 29]. Therefore, the absorption spectrum of microparticles in plastic samples can be accepted as the black body absorption spectrum. In the numerical investigations, both light-absorption and light-scattering spectra of microparticles should be taken into account [28]. However, it is considered that light-absorbing microparticles in transparent polymers become such optical inhomogeneities which increase the absorption of laser radiation that causes drastic heating of the vicinity of the focal region and thus, changes of the chemical composition in polymers can be occurred up to formation of a burnt area [18-21].

Fig. 5 shows marks of different sizes obtained in polystyrene containing light-absorbing carbon microparticles under irradiation with single pulses of a YAG:Nd laser $(\lambda=$ $532 \mathrm{~nm}, \tau=7 \mathrm{~ns})$ with different energies (1-3) and number of pulses (4-6). No marks are observed at $E<0.64 \mathrm{~mJ}$.

Microparticles suspended in the transparent polymer are heated under focused laser irradiation up to a temperature of several thousands of Kelvin. In the vicinity of laserheated microparticles, dark marks are created as result of pyrolysis of the polymer matrix. The occurrence of dark centers has a threshold nature, i.e. there is a minimum laser energy $E_{\text {th }}$ at which marks are formed. The threshold laser pulse energy depends on the sizes and concentration (density) of microparticles in polystyrene [19-21].
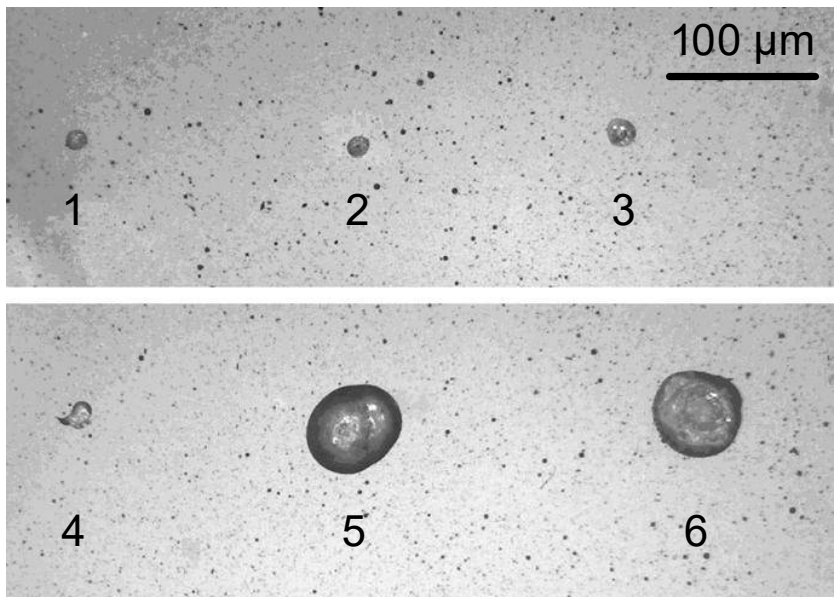

Fig. 5 Micrographs of laser-induced marks formed in polystyrene, activated by light-absorbing carbon microparticles, at irradiation with a single pulse (1-3) and 10 pulses (4-6), respectively with energy $E(\mathrm{~mJ}): 1.9(1,4), 3.7(2,5), 6.8(3,6)$.

In fact, that transparent polystyrene suspensions of carbon or another light-absorbing microparticles can be applied as a thin film on different objects. Laser-induced creation of marks in the volume of such polymers is an efficient technique for digital recording and thus it is possible to provide marking and supply relevant information for any object and item which is coated with that polymer film.

Fig. 6 shows solidified polymer film (thickness $\sim 0.2$ $\mathrm{mm}$ ) with dark marks of different size which were created in the volume of the film under irradiation with different number of pulses $(\tau=20 \mathrm{~ns})$ of the first harmonic $(\lambda=1064$ $\mathrm{nm})$ of a YAG:Nd laser with the same energy $(E=35 \mathrm{~mJ})$.

$\begin{array}{lllllllllll}1 \mathrm{~mm} & 16 & 18 & 20 & 22 & 25 & 30 & 40 & 50 \\ 13 \quad 14 & 15 & 17 & 19 & 21 & 23 & 27 & 36 & 45\end{array}$

Fig. 6 Micrograph of the marking area of the polymer film with light-absorbing microparticles showing the marks obtained as result of irradiation with different number of nanosecond laser pulses of the same energy. The numbers in the figure correspond to the number of laser shots. 
By varying laser energies, number of pulses and employing focusing lenses with different shapes and focal lengths, it is possible to obtain marking centers with various linear sizes and shapes (e.g. concentric circles of different diameters, ellipses, ellipsoids, spheres, bubbles, etc.) located at different depths. This can be used for laserinduced creation of digitally recording areas in the bulk and subsurface regions of polystyrene and other materials.

\section{Laser-induced light-emitting marking centers in or- ganic materials with photo-initiated compounds}

In conventional optical recording methods, crosstalk or interference of different recording components (marks) and layers often leads to reduced recording densities. It is possible, however, to improve the spatial resolution and thus the density of digital optical recording by using laserinduced marks which act as light emission centers under backlight illumination [22-27]. The most promising materials for such a purpose are those in which a local change in the complex refractive index, structure or spectralluminescent properties occurs in result of multi-photon absorption of ultrashort pulses of recording radiation.

Emitting centers can thus be obtained in thermally stable photosensitive organic chromone-based materials in selected layers by two-photon absorption of laser radiation with a wavelength that is not absorbed by the polymer matrix and chromone molecules [22-24]. Separate organic molecules in the laser focal area change their structure because of two-photon absorption and they become fluorescent marks. The density of created marks depends on the energy and number of recording laser pulses. The distance between the neighboring emitting centers created in the polymer matrix with embedded chromone molecules by short laser pulses $(\lambda=532 \mathrm{~nm}, \tau=0.8 \mathrm{~ns})$ is about $5 \mu \mathrm{m}$ and mark sizes are less than $2 \mu \mathrm{m}$ (Fig. 7). The brighter marks were created by increasing the number of recording laser pulses.

The different number of laser pulses incident to the local area of a given mark leads to creation of different number of induced fluorescent centers and thus to different brightness level. Fluorescent marks with specific intensities can therefore be created by multi-shot laser irradiation and careful selection of the applied number of laser pulses. The resulting light emitting marks can consequently be read and classified in respect to their brightness by standard photodetection methods [24]. Since even a single molecule of chromone can serve as a fluorescent center and the waveguide layer thickness is limited by the reading radiation wavelength, the maximum recording density depends only on the recording laser beam caustic parameters

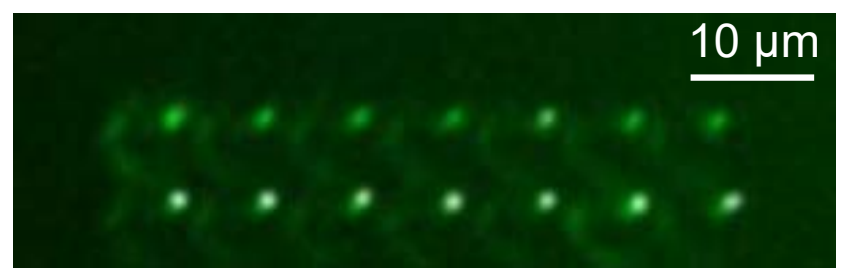

Fig. 7 Micrograph of laser-induced fluorescent marks formed in the volume of an organic chromone-based material by irradiation with single pulses of a YAG:Nd laser $(\lambda=532 \mathrm{~nm}, \tau=0.8 \mathrm{~ns}$.). Brighter marks are obtained by increasing the number of recording laser pulses.
Embedded information can in fact be read by injection of UV radiation into waveguide layers located between the photosensitive layers. In such a case the UV radiation partially penetrates into the adjacent layers with recorded marks and excites light emission from them due to fluorescence. Given that even a single chromone molecule can act as a fluorescent center and that the thickness of a waveguide layer can be as low as the reading radiation wavelength, the maximum recording density and spatial resolution depend only on the caustic of the recording laser beam and can closely approach the known theoretical limits.

The recording carried out by multi-photon excitation of organic molecules (photo-chromogenic chromones) and transition of them to a thermo-stable fluorescent state is much effective compared with another processes based on photoluminescence or refractive index change [25-27].

\section{Conclusion}

The development of laser-based techniques for creation of geometrically parameterized, color-enhanced, and lightemitting marking centers in different materials opens new opportunities for image formation, volumetric optical marking and digital recording in various physical objects. It is found that while studies on timing and energy characteristics of laser pulses and irradiation conditions are of primary importance for the creation of parameterized marks with different geometries, the research and selection of appropriate materials (commonly transparent dielectrics) play a key role in the marking with colored and light emitting centers. It is practically important to continue the experimental work with organic materials transparent to selected laser wavelengths and investigate interaction of short laser pulses with polymers including study of the photoluminescent and nonlinear optical properties of organic chromonebased materials as a promising recording medium.

\section{Acknowledgments}

A part of this work was carried out under the Cooperative Research Project (No H27-45, 2015) of the Research Institute of Electronics, Shizuoka University. The authors are also grateful to Professor Kamen Kanev (Shizuoka University, Japan) for useful discussion of the results.

\section{References}

[1] R. M. Wood: "Laser-Induced Damage of Optical Materials" (IOP Publishing, Bristol, 2003) p. 241.

[2] C. W. Carr, H. B. Radousky, A. M. Rubenchik, M. D. Feit and S. G. Demos: Phys. Rev. Lett., 92, (2004) 087401.

[3] E. G. Gamaly, S. Juodkzis, K. Nishimura, H. Misawa, B. Luther-Davies, L. Hallo, P. Nicolai and V. T. Tikhonchuk: Phys. Rev. B, 73, (2006) 214101.

[4] A. A. Manenkov: Proc. SPIE, 7132, (2008) 713202.

[5] V. A. Gnatyuk: Physica B: Condens. Mater., 308-310, (2001) 935.

[6] J. Han, Y. Li, T. Duan, C. He, Ya Gao, G. Feng and L. Yang: Optik - Intern. J. for Light and Electron Optics, 124, (2013) 1940.

[7] I. Troitski: U.S. Patent 6768080 (2004).

[8] I. Troitski: Proc. SPIE, 5664, (2005) 293.

[9] V. A. Gnatyuk, K. Kanev and S. V. Gagarsky: J. Adv. Res. Phys., 2, (2011) 021101. 
[10] V. A. Gnatyuk, K. D. Kanev, V. Mizeikis, T. Aoki, S. V. Gagarsky and L. V. Poperenko: Proc. $11^{\text {th }}$ Int. Conf. on Global Research and Education in Engineers for Better Life. Inter-Academia, Budapest, (2012) p. 506.

[11] V. A. Gnatyuk, O. I. Vlasenko, S. N. Levytskyi, K. S. Zelenska, L. V. Poperenko, S. V. Gagarsky and T. Aoki: Photonics Technol., 2014 Fotonica AEIT Italian Conf., (2014) DOI: 10.1109/Fotonica.2014.6843885.

[12]D. Paipulas, V. Kudriašov M. Malinauskas, V. Smilgevicius and V. Sirutkaitis: Appl. Phys. A, 104, (2011) 769.

[13]L. Skuja, H. Hosono and M. Hirano: Proc. SPIE, 4347 (2000) 155.

[14] G. Ligbado, A. Horn, E. W. Kreutz, M. M. Krauss, N. Siedow and H. Hensel: Proc. SPIE, 5989, (2005) $59890 \mathrm{~K}$

[15]I. Troitski: Proc. of SPIE, 5273, (2004) 192.

[16] J. B. Lonzaga, S. M. Avanesyan, S. C. Langford and J. T. Dickinson: J. Appl. Phys., 94, (2003) 4332.

[17] T. Hongo, K. Sugioka, H. Niino, Ya. Cheng, M. Masuda, I. Miyamoto, H. Takai and K. Midorikawa: J. Appl. Phys., 97, (2005) 063517.

[18] K. Kanev, V. Mizeikis and V. Gnatyuk: Proc. Joint Int. Conf. on Human-Centered Computer Environments, Aizu-Wakamatsu, (2012) p. 251.

[19] S. E. Zelensky, O. V. Kopyshinsky, V. V. Garashchenko, A. S. Kolesnik, V. M. Stadnytskyi, K. S. Zelenska and Ye. V. Shynkarenko: Semicond. Phys., Quant. Electron. \& Optoelectron., 13, (2010) 70.
[20] S. E. Zelensky, A. S. Kolesnik, A. V. Kopyshinsky, V. V. Garashchenko, K. S. Zelenska, V. M. Stadnytsky and E. V. Shinkarenko: Ukr. J. Phys., 54, (2009) 983.

[21] K. S. Zelenska, S. E. Zelensky, L.V. Poperenko, K. Kanev, V. Mizeikis and V. A. Gnatyuk: Opt. Laser Technol, 76, (2016) 96.

[22] A. Ayt, V. A.; Barachevsky, O. I. Kobeleva, T. M. Valova, S. V. Gagarskiy, V. V. Kiyko, A. N. Sergeev, A. V. Veniaminov, V. V. Zakharov, M. Krayushkin and H. Iglev: Laser Optics, 2014 Int. Conf., Saint Petersburg, (2014) DOI: 10.1109/LO.2014.6886252.

[23] V. A. Barachevsky, M. M. Krayushkin, V. V. Kyiko and E. P. Grebennikov: Phys. Status Solidi C, 8, (2011) 2841.

[24] V. Kurbangaev, N. Poroshin, P. Malushev and P. Shmelin: Photonica, 2, (2012) 74 (in Russian).

[25] E. N. Glezer, M. Milosavljevic, L. Huang, R. J. Finlay, T.-H. Her, J. P. Callan and E. Mazur: Opt. Lett., 21, (1996) 2023

[26]Z. G. Nie, K. S. Lim, W. Y. Jang, H. Y. Lee, M. K. Lee and T. Kabayashi: J. Phys. D: Appl. Phys., 43, (2010) 485101.

[27]. K. L. N. Deepak, R. Kuladeep, S. Venugopal Rao, and D. Narayana Rao: Chem. Phys. Lett., 503, (2011) 57.

[28] V. A. Gnatyuk., T. Aoki and Y. Hatanaka: Appl. Surf. Sci., 216, (2003) 88.

[29] R. W. Bergstrom, Ph. B. Russell and Ph. Hignett: J. Atmos. Sci. 59, (2002) 567.

(Received: May 27, 2015, Accepted: April 1, 2016) 